Timon Hitz, Matthias Heinen, Jadran Vrabec, Claus-Dieter Munz

\title{
Comparison of macro- and microscopic solutions of the Riemann problem I. Supercritical shock tube and expansion into vacuum
}

Journal article | Accepted manuscript (Postprint)

This version is available at https://doi.org/10.14279/depositonce-9565

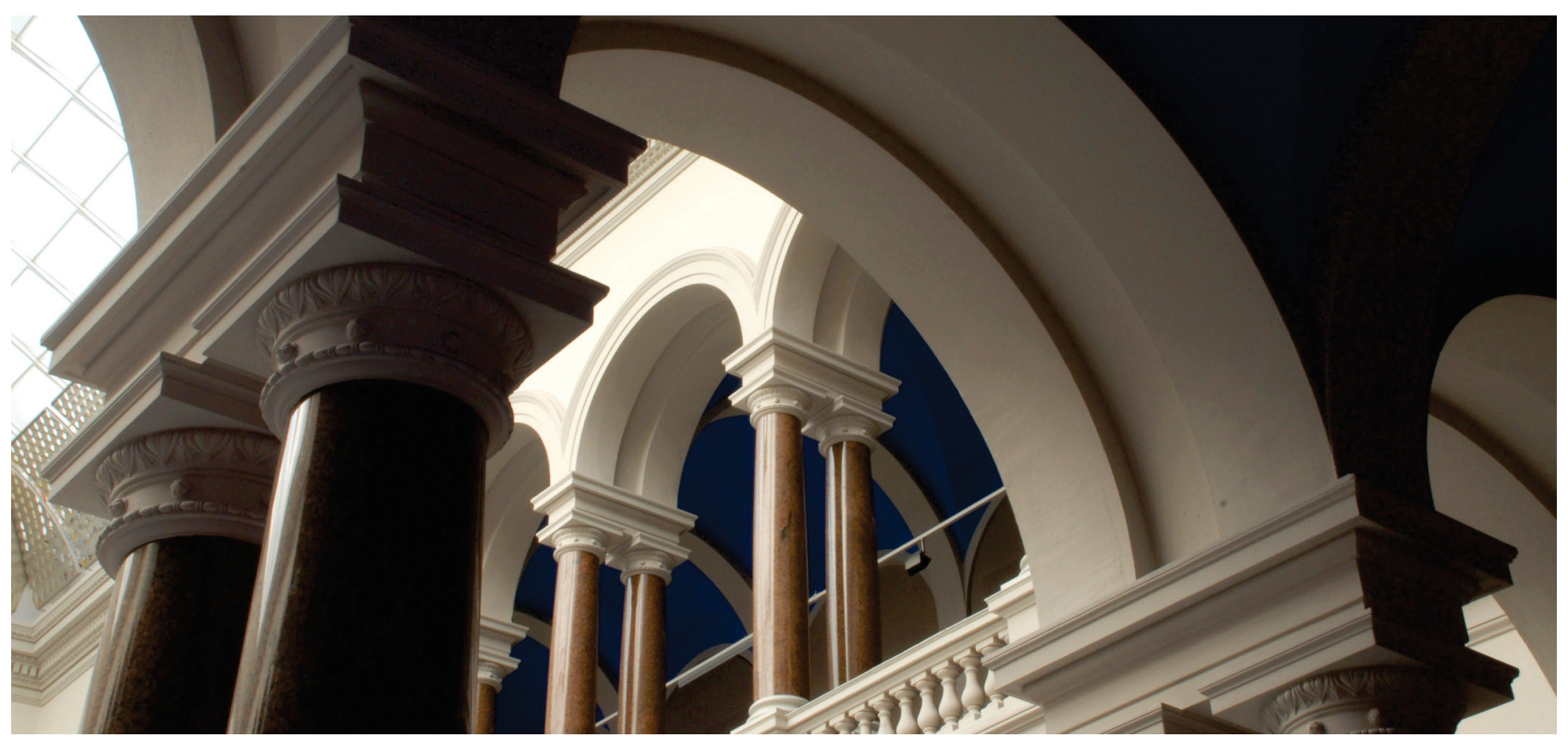

Hitz, T., Heinen, M., Vrabec, J., \& Munz, C.-D. (2020). Comparison of macro- and microscopic solutions of the Riemann problem I. Supercritical shock tube and expansion into vacuum. Journal of Computational Physics, 402, 109077. https://doi.org/10.1016/j.jcp.2019.109077 
Accepted manuscript of: Hitz, T., Heinen, M., Vrabec, J., \& Munz, C.-D. (2020). Comparison of macroand microscopic solutions of the Riemann problem I. Supercritical shock tube and expansion into vacuum. Journal of Computational Physics, 402, 109077. https://doi.org/10.1016/j.jcp.2019.109077

(C) 2019 This manuscript version is made available under the CC-BY-NC-ND 4.0 license https://creativecommons.org/licenses/by-nc-nd/4.0/

\title{
Comparison of Macro- and Microscopic Solutions of the Riemann Problem I. Supercritical Shock Tube and Expansion into Vacuum
}

\author{
Timon Hitz ${ }^{\mathrm{a}}$, Matthias Heinen ${ }^{\mathrm{b}}$, Jadran Vrabec ${ }^{\mathrm{b}}$, Claus-Dieter Munz ${ }^{\mathrm{a}}$ \\ ${ }^{a}$ Institute of Aerodynamics and Gasdynamics, University of Stuttgart, 70550 Stuttgart, \\ Germany \\ ${ }^{b}$ Thermodynamics and Process Engineering, Technical University of Berlin, 10623 \\ Berlin, Germany
}

\begin{abstract}
The Riemann problem is a fundamental concept in the development of numerical methods for the macroscopic flow equations. It allows the resolution of discontinuities in the solution, such as shock waves, and provides a powerful tool for the construction of numerical flux functions. A natural extension of the Riemann problem involves two phases, a liquid and a vapour phase which undergo phase change at the material boundary. For this problem, we aim at a comparison with the macroscopic solution from molecular dynamics simulations. In this work, as a first step, the macroscopic solution of two important Riemann problem scenarios, the supercritical shock tube and the expansion into vacuum, were compared to microscopic solutions produced by molecular dynamics simulations. High fidelity equations of state were used to accurately approximate the material behaviour of the model fluid. The results of both scenarios compare almost perfect with each other. During
\end{abstract}

Email address: hitz@iag.uni-stuttgart.de (Timon Hitz) 
the vacuum expansion, the fluid obtained a state of non-equilibrium, where the microscopic and macroscopic solutions start to diverge. A limiting case was shown, where liquid droplets appeared in the expansion fan, which was approximated by the macroscopic solution, assuming an undercooled vapour. Keywords:

Riemann Problem, Real Gas, Supercritical fluid, Vacuum Riemann

Problem, Finite Volume, Molecular Dynamics Simulation

\section{Introduction}

The Riemann problem poses an initial value problem for a conservation equation with piecewise constant initial data. In the numerical approximation of the compressible flow equations by finite volume (FV) schemes, it is one of the basic building blocks. Because the FV schemes approximate the integral conservation equations, they allow for discontinuities between the grid cells. In his pioneering work, Godunov [1] proposed to use the solution of the Riemann problem to determine the flux between the grid cells. Taking into account the information about the waves generated by a discontinuity establishes the robustness of such FV schemes, e.g., at shock waves or in under-resolved regions of compressible fluid flows. Later, several approximations of the solution of the Riemann problem have been developed, which preserve the advantageous properties of the Godunov scheme while reducing the computational effort. A comprehensive presentation of the Riemann problem and its approximations is given by Toro in his book [2].

The solution of the Riemann problem in gas dynamics is well-known under certain convexity constraints for the underlying equation of state (EOS). 
Here, the solution consists of four constant states that are separated by three waves. The outer waves are shock waves or rarefaction waves, associated with the characteristic wave speeds $v-c$ and $v+c$. The characteristic field of flow transport $v$ is linearly degenerate and exhibits a contact discontinuity, with a constant pressure and velocity across it. Menikoff and Plohr [3] describe wave structures that may occur if the standard convexity conditions are not satisfied. Beside the classical waves, shock wave, rarefaction wave and contact discontinuity, anomalous entities such as splitted waves or composite waves may form. Near a phase transition, an additional wave that corresponds to the phase interface appears in the wave structure of the Riemann problem. This is possible because the hyperbolicity of the Euler equations breaks down in the spinodal region of the state space and imaginary eigenvalues occur. To avoid such non-physical states in the macroscopic solution, information from the local thermodynamic behavior is needed and has to be incorporated into the macroscopic solution of the Riemann problem. Some additional information about local thermodynamic processes has to be formulated. One approach is based on a so-called kinetic relation that controls the mass transfer across the phase interface (see, e.g. LeFloch [4]). But the situation is not fully clear up to now and even more unclear is the numerical modelling.

In the last decades, molecular dynamics simulations became more powerful due to advanced algorithms and increasing computer efficiency. They resolve matter down to the atomistic scale by treating every molecule individually as a mechanical object. A central role is being played by the force field that describes the intermolecular interactions, like dispersion or repulsion due to Pauli exclusion. Once assigned to the molecules, these interactions 
not only determine their individual trajectories under given boundary conditions, but also contain, for a molecular ensemble, the entire set of thermodynamic properties in a consistent manner. MD has a sound physical basis and is thus a versatile approach that can be applied to a wide range of problems, like adsorption, diffusion or evaporation. However, a drawback is the associated computational effort. Newton's equations of motion must be solved numerically with a time step on the order of femtoseconds and the spatial resolution is on the Angström scale so that studying macroscopic processes is challenging.

The objective of our research activities is to compare the macroscopic modelling and the microscopic thermodynamic behavior. Hence, we compare molecular dynamics (MD) simulations for Riemann problem scenarios with macroscopic solutions. The most interesting situations are those, of course, in which phase transitions occur and the macroscopic solutions depend on the local thermodynamic modelling. To allow for a full correspondence of the macro- and microscale solutions, the present MD simulations were based on the Lennard-Jones model fluid with a truncated and shifted potential (LJTS). For this model fluid, two highly accurate equations of states for the macroscopic simulations are available: the empirical EOS by Thol et al. [5] and the semi-empirical EOS by Heier et al. [6], which have similar mathematical properties as the van der Waals EOS. This gives the chance to directly compare two topics. First, the macroscopic and the microscopic Riemann problem solutions directly and then the molecular dynamics Riemann problem solution with results by a finite volume method on some appropriate grid. We start here with a validation of this approach and consider Riemann 
problems in the supercritical regime without phase transition. The expansion of a fluid into vacuum leads to simple simulation conditions for MD. Hence, this is our second class of benchmark problems. Here, we see the following: if the initial conditions are such that due to the strong pressure drop in the rarefaction fan, liquid droplets appear in MD simulations, then the macroscopic simulations become more difficult to obtain due to instabilities. We assume that we have to change the modelling of the macroscopic Riemann problem solution and have to take phase transition in our numerical flux calculation into account.

We restrict ourselves in this first paper to the description of the methods and the validation of this approach. The interesting topic of the approximation of phase transition in macroscopic simulations is only touched. Our research activities are embedded in the DFG collaborative research center SFB-TRR 75 "Droplets under extreme ambient conditions". Hence, we have the possibility to continue this research in the coming years, especially looking at situations, in which phase transitions do occur. MD results for these situations will be documented and compared with macroscopic simulations that have to take phase transitions appropriately into account.

The structure of the paper is as follows. In Section 2, the continuum equations, the EOS and the Riemann problem are introduced. The numerical methods are discussed in Section 3. Results for a supercritical shock tube Riemann problem and several vacuum Riemann problem scenarios are given in Section 4, followed by a conclusion in Section 5 . 


\section{Theory}

\subsection{Equations of the Continuum System}

The compressible Euler equations in one spatial dimension are written in conservation form as

$$
\mathbf{U}_{t}+\mathbf{F}(\mathbf{U})_{x}=0
$$

with

$$
\mathbf{U}=\left(\begin{array}{c}
\rho \\
\rho v \\
\rho e
\end{array}\right), \quad \mathbf{F}=\left(\begin{array}{c}
\rho v \\
\rho v^{2}+p \\
v(\rho e+p)
\end{array}\right)
$$

The variables are density $\rho$, velocity $v$ and specific total energy $e=\epsilon+\frac{1}{2} v^{2}$, which is composed of the internal part $\epsilon$ and the kinetic part $\frac{1}{2} v^{2}$. In addition to the conservative state vector $\mathbf{U}$, the primitive variables can be written as a state vector $\mathbf{W}=(\rho, v, p)^{\top}$, where $p$ denotes the thermodynamic pressure. An EOS is required as a closure relation between the variables pressure, density and internal energy

$$
\epsilon=\epsilon(\rho, p) ; \quad p=p(\rho, \epsilon)
$$

In this study, we consider the Lennard-Jones model fluid with a truncated and shifted potential (LJTS). Two highly accurate EOS are available in this case: The empirical EOS by Thol et al. [5] and the semi-empirical EOS by Heier et al. [6]. Both equations are fundamental EOS in terms of the reduced Helmholtz free energy $\alpha$. It can be decomposed into the contribution of a monatomic ideal gas, $\alpha^{0}$ and a residual part, $\alpha^{\mathrm{r}}$. Note that the superscript ${ }^{0}$ always refers to a state described by the ideal gas law of a monatomic gas. The Helmholtz free energy is given as a function of reduced density $\delta=\rho / \rho_{\mathrm{c}}$ 
and inverse reduced temperature $\tau=T_{\mathrm{c}} / T$, where the subscript ${ }_{\mathrm{c}}$ indicates the critical point,

$$
\frac{a(\rho, T)}{R T}=\alpha(\delta, \tau)=\alpha^{0}(\delta, \tau)+\alpha^{\mathrm{r}}(\delta, \tau)
$$

Using derivatives of Eq. (4), any thermodynamic quantity of interest can be obtained directly. An overview of the necessary relations is provided in $[7,8]$. Since these fundamental EOS are given in terms of density and temperature, the closure condition for the Euler equations, Eq. (3), is not available in a closed form. For that purpose, a Newton-Raphson method was used to calculate the temperature iteratively for a given density and internal energy, provided by the CFD method. With known density and temperature, all other thermodynamic quantities can be calculated.

The EOS used in this study apply two different approaches expressing the residual part $\alpha^{\mathrm{r}}$. Thol et al. [5] provided a multiparameter EOS using the framework of Span [9]. Here, the residual Helmholtz energy was described by a sum of polynomial, exponential and Gaussian terms. The coefficients were determined by a fitting procedure on the basis of reference data from molecular dynamics simulations. This model is referred to as LJTS EOS in this study. It is in excellent agreement with reference data, but the evaluation of Eq. (4) is computationally costly. Furthermore, the occurrence of multiple Van-der-Waals loops in the two-phase region may cause difficulties for root finding algorithms. The PeTS EOS by Heier et al. [6] considers a framework based on perturbation theory [10]. The residual Helmholtz energy was split into the contribution of the reference potential and the contribution due to perturbation. Compared to the LJTS EOS, its evaluation is computationally cheaper and the presence of a single Van-der-Waals loop simplifies the usage 
of root finding algorithms. However, the PeTS EOS is somewhat less accurate. Both EOS provide formulations $[7,8]$ to calculate the thermodynamic properties pressure $p$, internal energy $\epsilon$ and speed of sound $c$.

To handle the transition to vacuum, the asymptotic limits of the Helmholtz energy for vanishing reduced density and reduced temperature, $\delta \rightarrow 0$ and $\tau \rightarrow+\infty$, have to be considered. In this case, the residual Helmholtz energy of both EOS approaches zero

$$
\lim _{\substack{\delta \rightarrow 0 \\ \tau \rightarrow+\infty}} \alpha^{\mathrm{r}}=0
$$

and only the perfect gas contribution remains. Both EOS use the same formulation for this perfect gas contribution $\alpha^{0}$ corresponding to a monatomic gas with an isobaric heat capacity $c_{p}^{0} / k_{\mathrm{B}}=2.5$, where $k_{\mathrm{B}}$ is the Boltzman constant. The perfect gas contribution reads as

$$
\alpha^{0}(\delta, \tau)=\ln \delta+1.5 \ln \tau+c_{1} \tau+c_{2}
$$

The coefficients $c_{1}, c_{2}$ were specified by Thol et al. [5] and Heier et al. [6] such that the perfect gas contributions to enthalpy and entropy vanish $h_{0}^{0}=s_{0}^{0}=0$ at the reference state $\left(T_{0}, p_{0}\right)=(0.8,0.001)$,

$$
c_{1}=-\frac{2.5}{\tau_{0}} \quad \text { and } \quad c_{2}=1.5-\ln \delta_{0}-1.5 \ln \tau_{0} .
$$

The asymptotic limits for pressure and internal energy are

$$
\begin{aligned}
& \lim _{\substack{\delta \rightarrow 0 \\
\tau \rightarrow+\infty}} p=\lim _{\substack{\delta \rightarrow 0 \\
\tau \rightarrow+\infty}} \rho R T\left(1+\delta \alpha_{\delta}^{\mathrm{r}}\right)=0, \\
& \lim _{\substack{\delta \rightarrow 0 \\
\tau \rightarrow+\infty}} \epsilon=\lim _{\substack{\delta \rightarrow 0 \\
\tau \rightarrow+\infty}} R T\left(\tau\left(\alpha_{\tau}^{0}+\alpha_{\tau}^{\mathrm{r}}\right)\right)=R T_{\mathrm{c}} c_{1} .
\end{aligned}
$$


The speed of sound $c$ is defined by

$$
c^{2}=\left(\frac{\partial p}{\partial \rho}\right)_{s}=\left(\frac{\partial p}{\partial \rho}\right)_{T}-\left(\frac{\partial p}{\partial T}\right)_{\rho}\left(\frac{\partial s}{\partial \rho}\right)_{T}\left(\frac{\partial s}{\partial T}\right)_{\rho}^{-1} .
$$

This formulation also falls back to the classical speed of sound of a perfect gas, which becomes zero for a vanishing temperature

$$
\lim _{\substack{\delta \rightarrow 0 \\ \tau \rightarrow+\infty}} c^{2}=\lim _{\substack{\delta \rightarrow 0 \\ \tau \rightarrow+\infty}} \frac{5}{3} R T=0 .
$$

\subsection{Riemann Problem}

The Euler equations (1) are a hyperbolic system of conservation laws with the real eigenvalues of the Jacobian of the flux

$$
\lambda_{1}=v-c, \quad \lambda_{2}=v, \quad \lambda_{3}=v+c .
$$

The initial value problem for the one-dimensional Euler equations, consisting of constant initial states, separated by a discontinuity at $x=0$, poses the Riemann problem

$$
\mathbf{U}(x, t=0)=\left\{\begin{array}{lll}
\mathbf{U}_{L} & \text { for } & x<0 \\
\mathbf{U}_{R} & \text { for } & x>0 .
\end{array}\right.
$$

Its solution is self-similar with respect to $x / t$ and consists of four constant states $\mathbf{U}_{L}, \mathbf{U}_{L}^{*}, \mathbf{U}_{R}^{*}, \mathbf{U}_{R}$, separated by elementary waves. The outer waves are either shock or rarefaction waves and the intermediate wave is a contact discontinuity. Each of the three wave types is attached to an eigenvalue of the system of equations. A typical situation is shown in the diagram in Fig. 1. The shock and rarefaction waves are non-linear waves and associated with $\lambda_{1,3}$, while the contact discontinuity is associated with $\lambda_{2}$. The latter is a 
linearly degenerate discontinuity in mechanical equilibrium, i.e. the velocity and pressure are equal on both sides. Consequently, there is no macroscopic mass flux across the contact discontinuity so that it can be considered as a material interface. The shock wave is a non-linear discontinuity where the Rankine-Hugoniot conditions apply. The rarefaction wave is a continuous solution where the so-called generalized Riemann invariants remain constant. As a consequence, the solution in a rarefaction wave is isentropic.

For the perfect gas, a solution has been proposed by Godunov [1] using a fixed point iteration. An extension to real gas EOS that remain smooth and convex has been introduced by Colella and Glaz [11] and further developed by Kamm [12]. The general approach is to find iteratively the velocity equilibrium across the contact discontinuity as a function of the unique pressure of the inner states via the Newton or the Secant method. Using this pressure, the inner states $\mathbf{U}_{L}^{*}$ and $\mathbf{U}_{R}^{*}$ are calculated by applying the relations across the outer waves, i.e., the Rankine Hugoniot conditions or the isentropic relations.

A special type of the Riemann problem is the shock tube problem, which is of elementary importance in gas dynamics. It describes two states in a tube that are separated by a membrane. A dense state under higher pressure is adjacent to a dilute state under low pressure. Both states are initially at rest. At time $t=0$, the membrane is removed and different waves propagate through the domain: A shock wave travels into the dilute fluid and a rarefaction wave travels into the dense fluid with the wave propagation speeds $S_{L}$ and $S_{R}$, respectively. Between these waves, two constant states appear that 
are separated by the contact discontinuity that travels with the speed $S^{*}$. The corresponding solution is shown in Fig. 1.

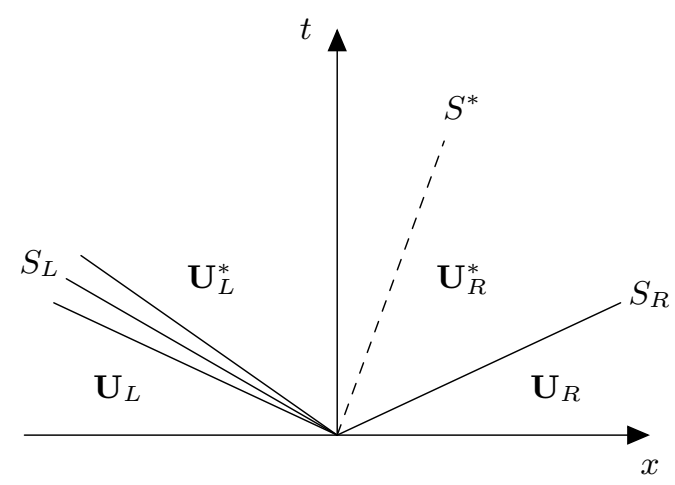

Figure 1: Solution structure for the shock tube problem where the high pressure is initially on the left and the low pressure on the right. Three waves divide four constant states: $\mathbf{U}_{L}, \mathbf{U}_{L}^{*}, \mathbf{U}_{R}^{*}, \mathbf{U}_{R}$.

Another special type of Riemann problem is the vacuum Riemann problem. In contrast to the shock tube problem, one of the states is a vacuum, i.e. $\mathbf{U}=(0,0,0)^{\top}$. Because the Euler equations are not valid in the vacuum, this scenario has to be considered as a free boundary problem, where the moving boundary represents the expansion front of the continuum phase into vacuum. A self-similar exact solution can be found for the perfect gas as shown by Halter and Martensen [13] and further discussed in Refs. [14, 15, 2].

If the vacuum is considered on the right side, the solution contains a single (left) rarefaction wave, cf. Fig. 2. The right wave vanishes and the contact discontinuity attaches the right boundary of the rarefaction wave. Integration of the generalized Riemann invariant across the rarefaction wave to the vacuum state yields a front speed of the expansion wave that is fully 
determined by the initial continuum state on the left

$$
S_{L}^{*}=v_{L}+\frac{2 c_{L}}{\gamma^{0}-1}
$$

where $\gamma^{0}=c_{p}^{0} / c_{V}^{0}$ is the isentropic coefficient of a monatomic ideal gas and $c_{p}^{0}$, $c_{V}^{0}$ are the heat capacities at constant pressure and volume of a monatomic ideal gas, respectively. Note that the wave speed $S_{L}^{*}$ describes the propagation speed of the outer most right wave of the left rarefaction wave, that lies adjacent to the star state, cf. Fig. 2. The solution reads as

$$
\mathbf{W}(x, t)=\left\{\begin{array}{lll}
\mathbf{W}_{L} & \text { if } \quad \frac{x}{t} \leq v_{L}-c_{L} \\
\mathbf{W}_{\text {fan }}=\left(\rho_{\mathrm{fan}}, v_{\mathrm{fan}}, p_{\mathrm{fan}}\right)^{\top} & \text { if } \quad v_{L}-c_{L}<\frac{x}{t}<S_{L}^{*} \\
\mathbf{W}_{R} & \text { if } \quad \frac{x}{t} \geq S_{L}^{*},
\end{array}\right.
$$

with

$$
\begin{aligned}
& \rho_{\text {fan }}=\rho_{L}\left(\frac{2}{\gamma^{0}+1}+\frac{\gamma^{0}-1}{\left(\gamma^{0}+1\right) c_{L}}\left(v_{L}-\frac{x}{t}\right)\right)^{\frac{2}{\gamma^{0}-1}} \\
& v_{\text {fan }}=\frac{2}{\gamma^{0}+1}\left(c_{L}+\frac{\gamma^{0}-1}{2} v_{L}+\frac{x}{t}\right) \\
& p_{\text {fan }}=p_{L}\left(\frac{2}{\gamma^{0}+1}+\frac{\gamma^{0}-1}{\left(\gamma^{0}+1\right) c_{L}}\left(v_{L}-\frac{x}{t}\right)\right)^{\frac{2 \gamma^{0}}{\gamma^{0}-1}}
\end{aligned}
$$

Figure 2 shows the solution structure of the vacuum Riemann problem.

The expansion of the continuum to the vacuum may be described as a free surface problem. For many physical problems, non-equilibrium effects have to be taken into account to model the physical phenomena correctly. In this case, a gas kinetic description has to be adopted. For our purpose here, the gas vacuum problem is an example for a single rarefaction wave, that can also be handled by molecular dynamics in an appropriate way. 


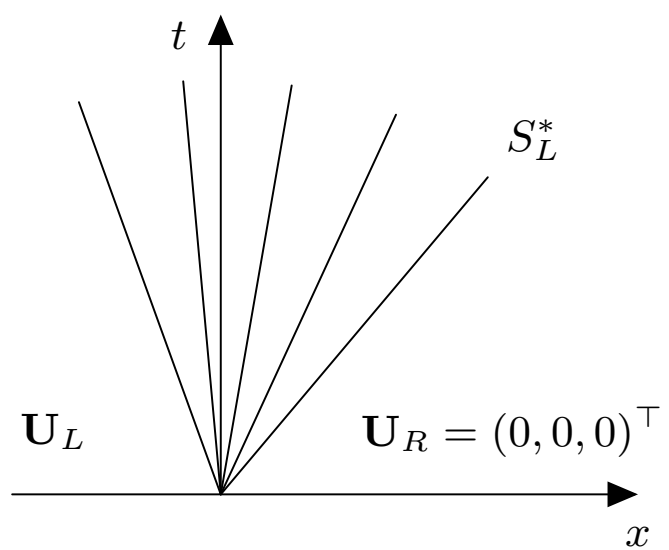

Figure 2: Solution structure of the vacuum Riemann problem, where the vacuum is located on the right. The left state is connected to the vacuum by a rarefaction wave, the contact discontinuity attaches the boundary of the rarefaction wave adjacent to the vacuum.

\section{Numerical Methods}

\subsection{Fluid-Solver FLEXI}

The conservation equations of the fluid equations are solved by a discontinuous Galerkin spectral element method (DGSEM), which is implemented as open source code FLEXI ${ }^{1}$. The numerical method is described by Hindenlang et al. [16] in detail. Hence, we only survey the basic building blocks to get some impression of the numerical method used. As usual in the discontinuous Galerkin approach, the solution and the fluxes are approximated by polynomials in each grid element allowing discontinuities between the elements. In DGSEM, the polynomial basis is a nodal one with Lagrangian polynomials defined by the Gauss points. We project the physical grid cell

\footnotetext{
${ }^{1}$ https://www.flexi-project.org/
} 
to a reference element and derive the weak formulation of the conservation equations in this reference element. The resulting volume and surface integrals are approximated by Gauss quadratures. As for Finite Volume schemes, numerical flux functions are needed for the coupling to the neighboring elements. If discontinuities appear in the solution, such as shock waves or material boundaries, the method is supplemented with a shock capturing method $[17,18]$, in which in elements with oscillations a total variation diminishing

finite volume method on sub-cells is activated. The indicator of Persson and Peraire [19] is used to detect oscillations. The structure of the DGSEM is kept - the local finite volume scheme on sub-cells may be interpreted as the alternative to evaluate the volume integral. The time integration method is explicit with high order Runge-Kutta schemes as proposed in [20].

\subsection{Numerical Flux Calculation}

The numerical flux solver for the vacuum Riemann problem is a little bit subtle not to produce negative internal energies and a break-up of the simulation. We apply here the Suliciu relaxation Riemann solver [21, 22] for the isentropic Euler equations which handles the vacuum state very well. The extension to the complete equations of gas dynamics has been described by Bouchut [23], based on a relaxation system for the Euler equations using a variety of supplementary variables such as pressure, speed of sound and entropy amongst others. If the underlying EOS is convex, an approximate Riemann solver can be constructed to solve the relaxation system and, by extension, the original Euler equations. To use a general EOS, the original method has 
been modified for this study by defining a local isentropic coefficient via

$$
\gamma_{\mathrm{loc}}=\frac{p}{\rho \epsilon}+1
$$

In case of a perfect gas, the local isentropic coefficient recovers the exact one: $\gamma_{\text {loc }}=\gamma^{0}=c_{p}^{0} / c_{V}^{0}=p / \rho \epsilon+1$. As shown in [23], the Suliciu solver preserves positivity of $\rho$ and $\epsilon$ and guarantees the inequalities of entropy as well as the maximum principle of entropy. We applied the numerical flux in flux difference form as proposed by Shen et al. [24]

$F=\frac{1}{2}\left(F_{L}+F_{R}\right)-\frac{1}{2}\left(\left|S_{L}\right|\left(\mathbf{U}_{L}^{*}-\mathbf{U}_{L}\right)+\left|S^{*}\right|\left(\mathbf{U}_{R}^{*}-\mathbf{U}_{L}^{*}\right)+\left|S_{R}\right|\left(\mathbf{U}_{R}-\mathbf{U}_{R}^{*}\right)\right)$,

in which the vacuum state can be directly inserted and the relaxation speeds remain bounded.

\subsection{Molecular Dynamics Simulations}

MD simulations were carried out with the open source code ls1 mardyn [25] that numerically solves Newton's equation of motion for large particle ensembles. Molecular interactions were assumed to be pairwise additive and evaluated explicitly within a specified cutoff radius $r_{c}$. In the present study, the LJTS fluid was simulated, being fully consistent with the EOS by Thol et al. [5] and Heier et al. [6]. This model has only two state-independent parameters for a given molecular species, i.e. for size $\sigma$ and dispersive energy $\epsilon$, and is very well suited to describe the thermodynamic properties of simple fluids, like the noble gases and methane $[26,27]$. It was selected since it offers additional advantages. First, the cutoff radius $r_{c}=2.5 \sigma$ is comparatively small, driving down computational costs. Second, long-range corrections can 
be omitted entirely because of the truncated potential definition. This is particularly convenient for inhomogeneous systems, where long range correction techniques are not straightforward.

\section{Results}

\subsection{Supercritical Shocktube Problem}

A shock tube scenario was defined for the LJTS fluid in the supercritical regime. The initial states were chosen such that one state lies in the liquid-like region and the other in the vapour-like region. A high temperature ensures that the solution remains in the supercritical regime. The initial data under consideration were

$$
(\rho, v, T)^{\top}= \begin{cases}(0.6,0.0,3.0)^{\top} & \text { if } x<x_{0} \\ (0.2,0.0,3.0)^{\top} & \text { if } x \geq x_{0} .\end{cases}
$$

For both equations of state used in this study, the initial pressures are calculated from the density and temperature to avoid errors by conversion comparing with the MD simulations, hence they differ in their values. E.g., for the LJTS EOS, the initial left pressure was $p=3.99$ and for the PeTS EOS it was $p=3.80$ while the right pressure was $p=0.657$ and $p=0.648$, respectively. This caused a shift in the solution for the pressure but achieved an improvement of the comparison of temperatures between MD and CFD data.

All physical properties were non-dimensionalized in terms of reference length $\sigma_{\text {ref }}=1 \AA$, reference energy $\epsilon_{\text {ref }} / k_{\mathrm{B}}=1 \mathrm{~K}$ and reference mass $m_{\text {ref }}=1 \mathrm{u}$. Consequently, the time reference is $t_{\text {ref }}=\sigma_{\text {ref }} \sqrt{m_{\text {ref }} / \epsilon_{\text {ref }}}$. In these 
reduced units, the critical point of the LJTS fluid is given by $\rho_{\mathrm{c}}=0.319$ and $T_{\mathrm{c}}=1.086$. The spatial domain was chosen as $[0,4000]$ and the initial discontinuity was located at the midpoint $x_{0}=2000$. The problem was simulated until $t_{\text {end }}=300.91$. For the CFD code FLEXI, the one-dimensional domain was discretized into 100 elements and a polynomial degree of $N=3$ was chosen that should result in a fourth order approximation. Discontinuities were detected with the Persson indicator [19] with threshold $\operatorname{Ind}_{\text {upper }}=-3$. In this case, the grid element was handled by switching to the local TVD finite volume scheme on the subgrid. The DG was applied again for $\operatorname{Ind}_{\text {lower }}=-5$. For time integration we used the explicit low storage fourth order Runge-Kutta scheme with five stages [20]. The stability criterion was chosen as CFL $=0.9$. In addition to the numerical simulation, an exact solution of the Riemann problem was calculated by the solution procedure of Kamm [12].

For the corresponding MD simulation, two homogeneous phases with a cuboid geometry were equilibrated at a temperature $T=3.0$, one with the higher density $\rho_{1}=0.6$ and one with the lower density $\rho_{2}=0.2$. They were merged such that physical contact between the phases was established through two planar interfaces, yielding a symmetric system, cf. Fig. 3. A few individual particles overlapping across at the interface were discarded. Periodic boundary conditions were established in all directions to mimic infinite extended interfaces and to avoid boundary effects in $x$ direction.

For symmetry reasons, the origin of the spatial coordinate $x$ was defined to coincide with the geometric center of the system so that only the right half of the system was considered in the following. Initially, the phases had an equal thickness of $\Delta x=2000$ and the initial interface position was 


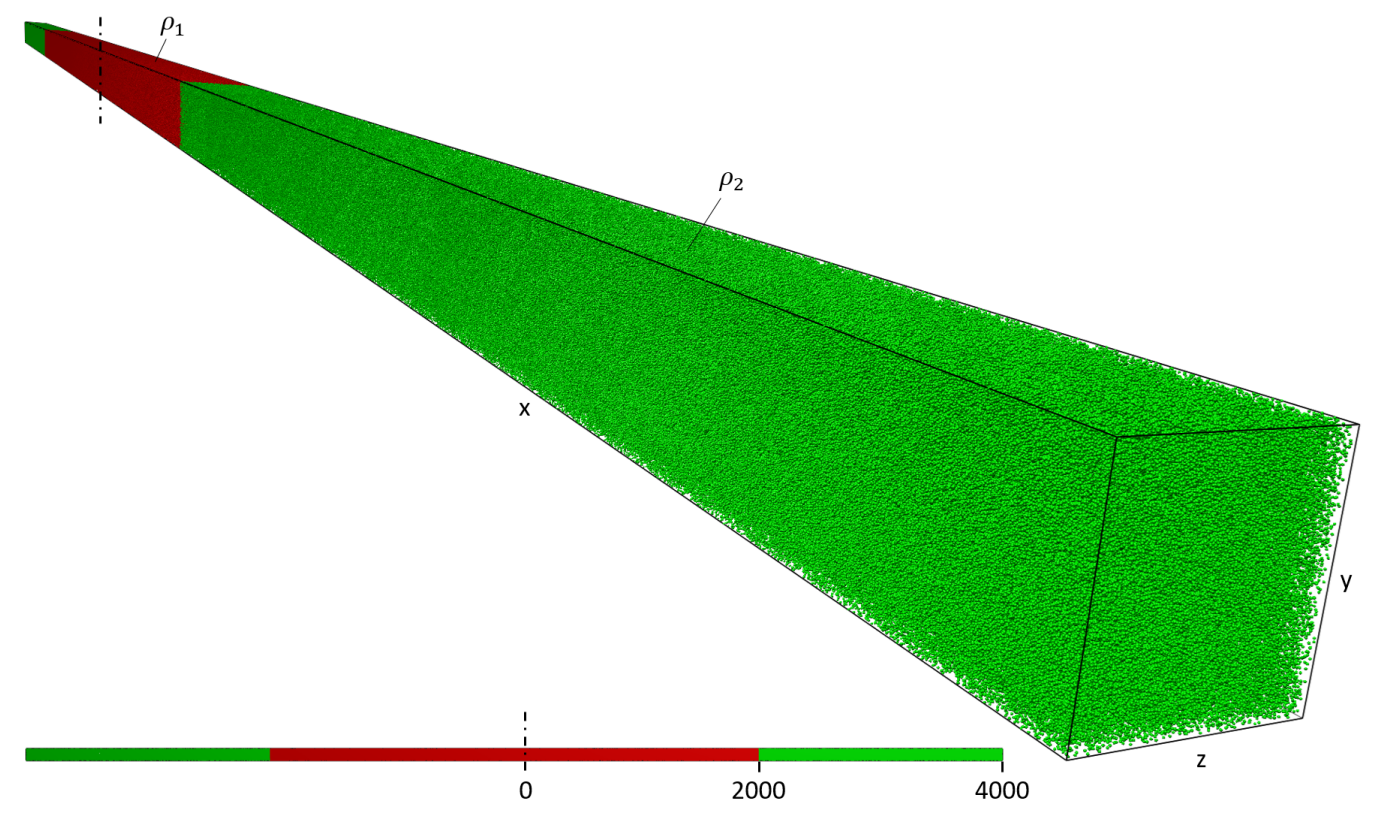

Figure 3: Snapshot of the shock tube scenario in a diagonal view to give an impression of the system on particle scale, and a plan view to clarify the system dimensions. The system was symmetric around $x=0$. Particles of the higher and lower density phase $\rho_{1}=0.6$ and $\rho_{2}=0.2$ were colored in red and green, respectively.

located at $x_{0}=2000$. The cross-sectional area was comparatively large $A_{y z}=\Delta y \Delta z=100^{2}$ to obtain good statistics while investigating a rapid process. Consequently, the system consisted of a large number of particles $N=3.2 \cdot 10^{7}$. Starting the simulation from the initial configuration, all individual particle trajectories were followed without any interventions, i.e., thermostating was avoided. Newton's equations of motion were solved numerically, employing the Leapfrog integrator that achieves a good energy conservation. Maintaining these conditions, the MD simulation results are directly comparable to the solution of the Riemann problem. To follow the temporal evolution of spatially resolved quantities, one-dimensional sampling 
was conducted to obtain density, temperature and hydrodynamic velocity profiles. For this purpose, the system was divided into bins with a thickness of $\delta=0.25$ and a volume of $\Delta V=\delta A_{y z}=2500$. The local density $\rho$ in a bin was calculated by

$$
\rho=\frac{N}{\Delta V}
$$

where $N$ is the number of particles in the bin. The velocity vector of the $j$-th particle is named $v_{j}$ and has the three components $v_{x, j}, v_{y, j}$ and $v_{z, j}$ into $x^{-}$, $y$-, and $z$-direction, respectively. The hydrodynamic velocity in $x$-direction $\hat{v}_{x}$ was obtained from the arithmetic mean of the velocity component $v_{x}$ of all particles in the bin

$$
\hat{v}_{x}=\frac{1}{N} \sum_{j=1}^{N} v_{x, j} .
$$

The temperature was sampled for the three spatial directions individually $T_{x}, T_{y}$ and $T_{z}$ because it cannot unconditionally be treated as a single scalar quantity under strong non-equilibrium conditions. The temperature is a measure for the kinetic energy of thermal motion only. Therefore, when accumulating the kinetic energy of the particle collective in a bin, the contribution due to hydrodynamic velocity has to be subtracted, yielding for the temperature in $x$ - direction

$$
k_{B} T_{x}=\frac{m}{3 N} \sum_{j=1}^{N}\left(v_{x, j}-\hat{v}_{x}\right)^{2}
$$

For the other two spatial directions, a vanishing hydrodynamic velocity can be assumed $\hat{v}_{y}=\hat{v}_{z}=0$, hence,

$$
k_{B} T_{y}=\frac{m}{3 N} \sum_{j=1}^{N} v_{y, j}^{2}
$$


applies, where $T_{z}$ is analogous. Since $T_{y}$ and $T_{z}$ were identical in the present scenario, they were summarized to a mean quantity

$$
T_{y z}=\left(T_{y}+T_{z}\right) / 2 .
$$

Quantities were averaged over a period of 5000 time steps, where a single time step $\Delta t$ corresponds to a physical time of $2 \mathrm{fs}$ for a fluid like Argon. This was a compromise for the sampling period being long enough to obtain good statistics, but short enough to ensure that the rapid changing profiles did not get blurred. The simulation was terminated when the system's reaction on the discontinuity was propagated to the system boundary, which was already the case after $2 \cdot 10^{5}$ time steps.

The results are shown in Fig. 4 for density, temperature, velocity and pressure. Each quantity was compared to MD simulation data, except for the pressure, which was not sampled in the MD simulations. The numerical results indicate that all properties are in excellent agreement with each other. The wave phenomena and the constant states in between are clearly identified: A shock wave propagates to the right, a rarefaction wave propagates to the left and a contact discontinuity is located in between. The approximate CFD solution adds some numerical diffusion, which smears out the shock and contact discontinuity compared to the exact solution. However, diffusion is also visible in the MD data, where viscous and diffusive effects are fully resolved. The shock capturing method was, as expected, only active around the shock wave, as indicated by the grey rectangle. Here, the method switches locally to a second order FV method with TVD reconstruction of the gradients on the sub-cells within the coarse DG grid element. Outside of this region, the solution was produced by the high order DG method. 

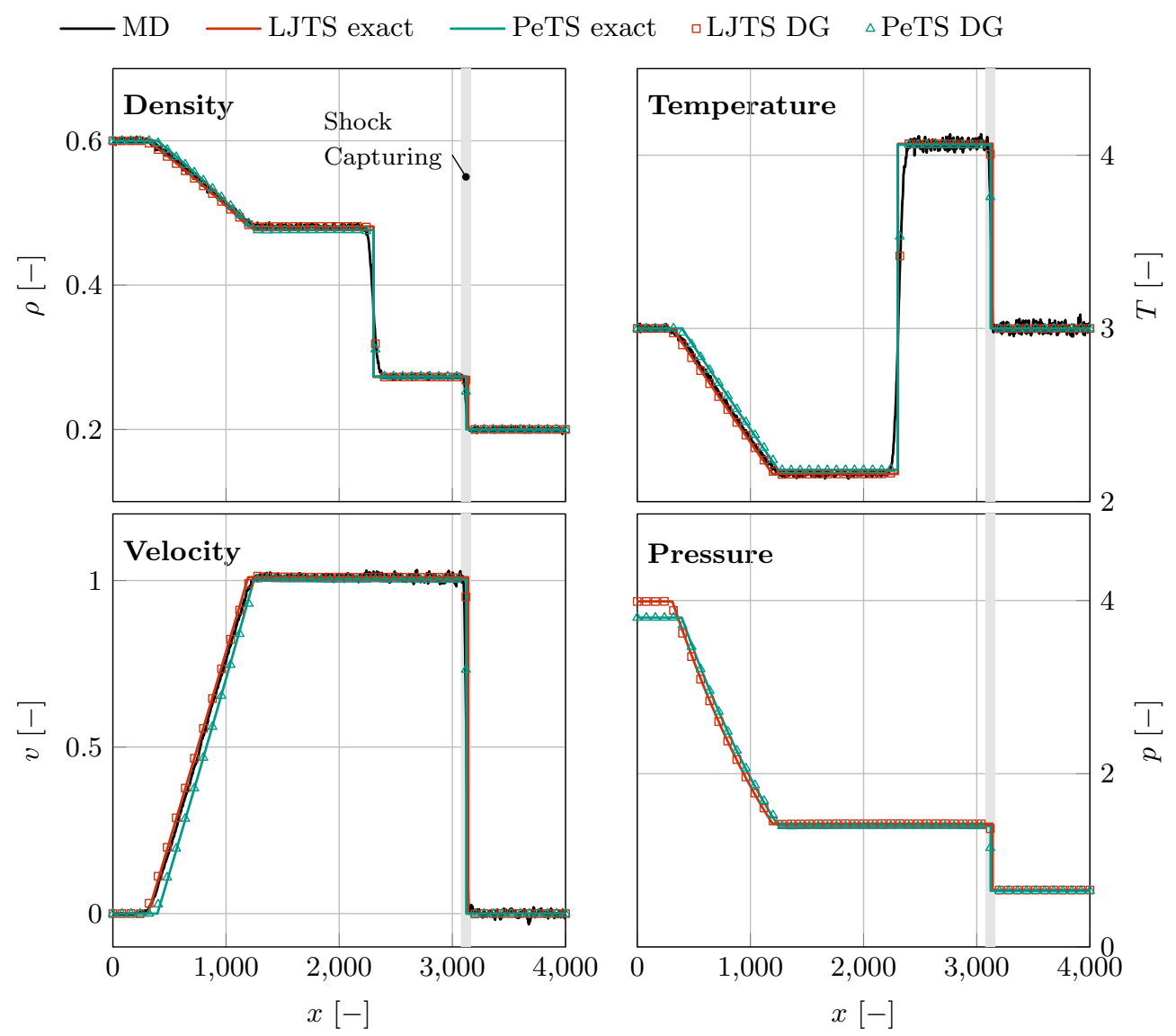

Figure 4: Results for the supercritical shocktube problem. The plot shows the results of the MD simulations, the exact solution of the corresponding macroscopic Riemann problem and the approximate solution by the DGSEM scheme using the Suliciu relaxation solver. The Riemann problem was solved resting either on the LJTS EOS or the PeTS EOS. The shock capturing was active in the region indicated by the grey rectangle.

Visible differences were observed for velocity and pressure in the comparison of the different EOS that were underlying to the CFD simulations. At the rarefaction wave, the PeTS EOS produces a slightly shifted position, whereas the LJTS EOS lies directly on the MD data. This effect can be at- 
tributed to an underestimation of the speed of sound by the PeTS EOS in the high density region as well as the different pressures of the initial datum. Similarly, the PeTS EOS underestimates the pressure in the high density region. Note that the pressure difference of the initial liquid states stems from the choice of initial values. To find the best basis for the comparison with MD simulation data, the temperature was chosen to be equal in the initial datum which caused the different plateaus in pressure.

Our conclusion with respect to this comparison of the numerical results is as follows. By using these corresponding thermodynamic relations of the LJTS EOS and the LJTS flow assumption in the MD simulations, we are able to get the same results by CFD and MD.

\subsection{Vacuum Expansion Problem}

As a first step to more subtle cases, we choose the so called vacuum Riemann problem, in which one state is the vacuum. The solution consists of a rarefaction wave to the vacuum with the attached contact discontinuity. The vacuum Riemann problem can be handled well by MD simulations. For the continuum equations, the transition to vacuum is a limit to the range of the validity of the equations and the approximation needs a flux calculation that keeps density and internal energy non-negative.

The vacuum expansion problem was considered for the LJTS fluid again and with the continuum phase on the left and the vacuum on the right. We investigate in the following four cases with a common temperature of $T_{L}=3.0$. The strength of the rarefaction wave was prescribed by a varying density. The baseline test case VP1 had an initial density $\rho_{L}=0.05$. The other cases had the densities: $\rho_{L}=0.01$ (VP2), $\rho_{L}=0.1$ (VP3) and $\rho_{L}=0.2$ 
(VP4). The full initial data of the vacuum Riemann initial were

$$
(\rho, v, T)^{\top}(x, 0)= \begin{cases}\left(\rho_{L}, 0.0, T_{L}\right)^{\top} & \text { if } x<x_{0} \\ (0.0,0.0,0.0)^{\top} & \text { if } x \geq x_{0} .\end{cases}
$$

The computational domain was chosen to be $x \in[0,4000]$ and the initial discontinuity was located at $x_{0}=2000$. In DG method we switched for these problems to the second order FV method with TVD limited gradient reconstruction, which is used as shock-capturing in sub-cells, to guarantee the positivity near the vacuum. The approximate Suliciu relaxation solver was used for the numerical flux function and time integration was done via an Euler explicit time integration with $\mathrm{CFL}=0.1$. At the vacuum domain boundary, the boundary values were extrapolated from the flow region as in a supersonic case. The domain was discretized into 400 elements. Both the LJTS and PeTS EOS, were alternatively used to obtain approximate solutions of the Riemann problem. Additionally, the exact solution for a perfect gas with $\gamma^{0}=1.667$ was computed, which corresponds to the perfect gas contribution of the LJTS model fluid.

For the MD simulations, exactly the same system geometry was used. Moreover, the simulation procedure and the sampling were conducted in the same way as described for the shock tube scenario with the left values of the density: $\rho_{L}=0.05,0.01,0.10,0.2$ and the replacement of the lower density phase by free space $\rho_{R}=0$ (vacuum). To maintain instant vacuum conditions, all particles that reached the system boundary at $x=4000$ were discarded, i.e. they left the computational domain. 


\subsubsection{Time Evolution of VP1}

Solutions of the time evolution of density, velocity and temperature for testcase VP1 at three times $t=91.18, t=182.37$ and $t=273.55$ are shown in Fig. 5. The structure of the exact solution is reproduced by our CFD
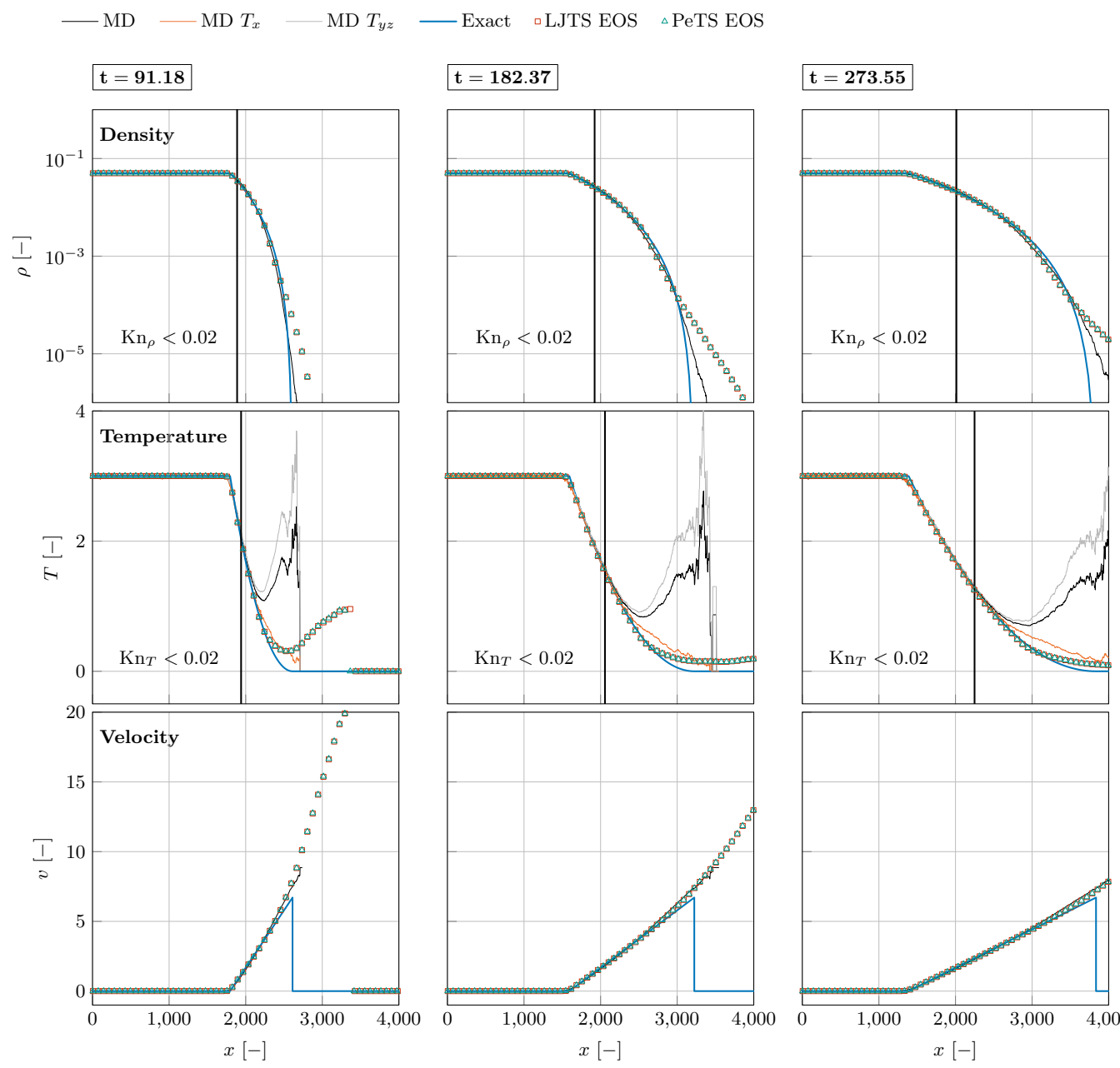

Figure 5: Results for the vacuum Riemann problem VP1 at time instances $t=91.18$, $t=182.37$ and $t=273.55$. The plot shows the approximate CFD solutions resting either on the LJTS or the PeTS EOS, an exact solution of the Riemann problem for the perfect gas law with $\gamma^{0}=1.667$ and MD simulation results. 
approximation as well as by MD simulation. The solution is determined by the rarefaction wave, which expands the gas isentropically to the vacuum. Note that the contact wave coincides with the tail of the rarefaction wave and is therefore not visible. At all time instances, depicted in Fig. 5, the agreement between all methods is excellent as long as the density is large enough. The LJTS and PeTS EOS show almost perfect agreement with each other for every variable, which is attributed to the identical perfect gas contribution that dominates the fluid behavior at low density.

The MD data were used to calculate the local Knudsen number to indicate the continuum breakdown. The Knudsen number is the ratio of the mean free path $\lambda$ and a characteristic length $L$ of the problem:

$$
\mathrm{Kn}=\frac{\lambda}{L} .
$$

In the present case, the choice of the characteristic length is not as clear. Therefore, we consider the local Knudsen number introduced by Boyd et al. [28]. It relies on the gradient of a physical state variable $\widetilde{\mathbf{W}}$, usually the density $\widetilde{\mathbf{W}}=\rho$ or temperature $\widetilde{\mathbf{W}}=T$, and reads as

$$
\mathrm{Kn}_{\widetilde{\mathbf{W}}}=\lambda \frac{|\nabla \widetilde{\mathbf{W}}|}{\widetilde{\mathbf{W}}} .
$$

An approximation of the mean free path $\lambda$ was given for a monatomic perfect gas in Bird [29] as

$$
\lambda=\frac{1}{\sqrt{2} \pi \rho \sigma^{2}},
$$

where $\sigma$ is the molecular diameter and $\rho$ the number density. Following Burt and Boyd [30], a flow can be considered to be a continuum if $\mathrm{Kn}_{\widetilde{\mathbf{w}}}<0.02$. To calculate the local Knudsen number, the MD data were smoothed using 
a moving average with a window size of 100. We considered the densitybased local Knudsen number $\mathrm{Kn}_{\rho}$ and the temperature-based local Knudsen number $\mathrm{Kn}_{T}$. The value $\mathrm{Kn}_{\rho, T}<0.02$ for the density-based and the temperature-based Knudsen number is plotted as a line parallel to the $x$ axes in the density and temperature plots, respectively. Both definitions of the local Knudsen number lead to similar values that indicate the location, at which the continuum assumption starts to fail.

Notable differences of the results for the different methods occur beyond the cut-off Knudsen number only. The density of all the methods approach zero. But the locations differ. The expansion to the vacuum by the DG scheme is the fastest one, followed by the MD results. The expansion of the exact perfect gas solution is the slowest one. This behavior is manifested also in the velocity plots that show strong deviations in the low density region. All results have the same gradient, but the front speeds differ. Such an artefact has also been observed by Bouchut [23]. The largest deviation was found for the temperature. While the exact perfect gas solution tends to a zero temperature, the DG scheme and the MD method do not. For the MD simulation the expected temperature is zero, as the number of particles strongly decrease towards the vacuum, which also decreases the interaction probability between particles. Consequently, a stronger deviation from the Maxwell distribution of the particles appears and the definition of a continuum temperature breaks down into an oscillatory behavior. Figure 5 also shows the temperatures that were calculated from the thermal motion of the particles in each spatial direction individually, cf. Eqs. (24) for $T_{x}$ and (26) for $T_{y z}$. 
Differences between both temperatures are visible which clearly indicate that a continuum temperature can not longer be defined.

\subsubsection{Convergence for VP1}

Because of the subtle behavior of the temperature approximation in the low density region, a convergence study for the DG scheme has been performed for the VP1 problem. VP1 was simulated based on the Suliciu numerical flux and the EOS of perfect gas with $\gamma^{0}=1.667$ on different grids ranging from 100 elements to 6400 elements. The left panel of Fig. 6 shows results for the temperature profile. Even with the EOS of perfect gas, a similar increase in temperature can be observed, but the temperature approaches zero as the mesh spacing is reduced. The convergence rate of the local maximum of the temperature in the higher Knudsen region is plotted in the right panel of Fig. 6. For an increasing number of elements, convergence is attained with a low rate of approximately 0.6 . The temperature increase obtained by the Suliciu flux is caused by different slopes of pressure and density. Considering the perfect gas law, the temperature was calculated by

$$
T=\frac{p}{\rho k_{\mathrm{B}}} .
$$

Near the vacuum, for very small values of density and pressure, the slopes of density and pressure are not identical. Beyond some threshold, the density gradient is smaller than the pressure gradient which, following Eq. (31), caused the non-physical temperature increase. Refining the grid this artefact disappears. This also appears in the simulations resting on the LJTS EOS and the PeTS EOS. In the high Knudsen regime, both EOS fall back to a perfect gas formulation. However, the implementation for both EOS still eval- 
uates Eq. (4) with a remaining contribution of the residual Helmholtz energy instead of Eq. (31). Consequently, the peak for the increasing temperature is reproduced differently than for the classical perfect gas law.
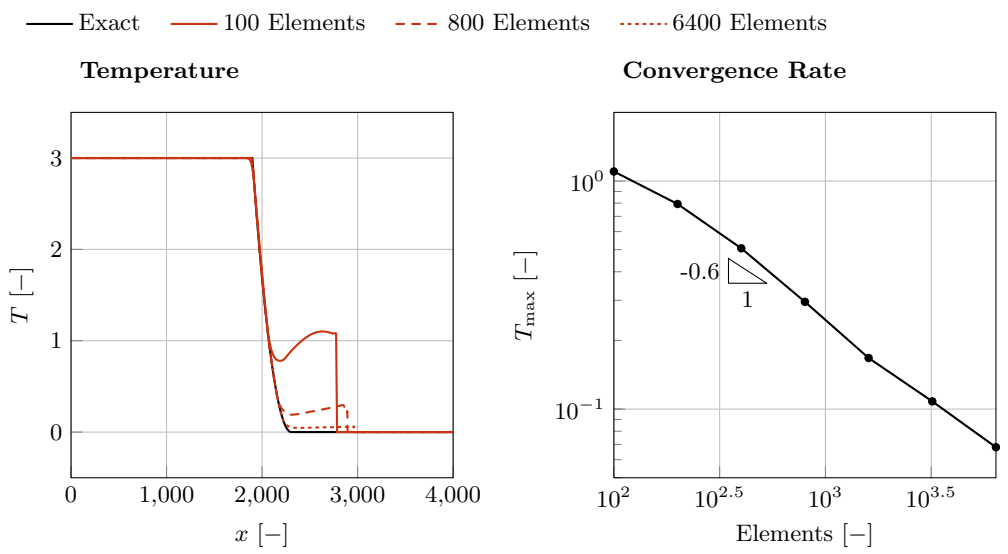

Figure 6: CFD convergence for the vacuum Riemann problem VP1 at the time instance $t=45.59$. The approximate solutions were obtained for the perfect gas with $\gamma^{0}=1.667$. Three grids are shown, constituted by 100,800 and 6400 elements. The data are compared to the exact solution.

Fig. 7 shows a comparison between the temperature profiles of two MD simulations with a different number of particles. The coarser simulation produced more scattering, but both simulations agree very well for small Knudsen numbers. In the high Knudsen number regime, the fine grid simulation shows a similar increase of temperature as the coarse grid simulation. Furthermore, the position of the local minimum is identical. Our explanation of this non-physical increase of the temperature is that non-equilibrium states affects again the averaging procedure for the mean temperature. 
- MD coarse — MD fine

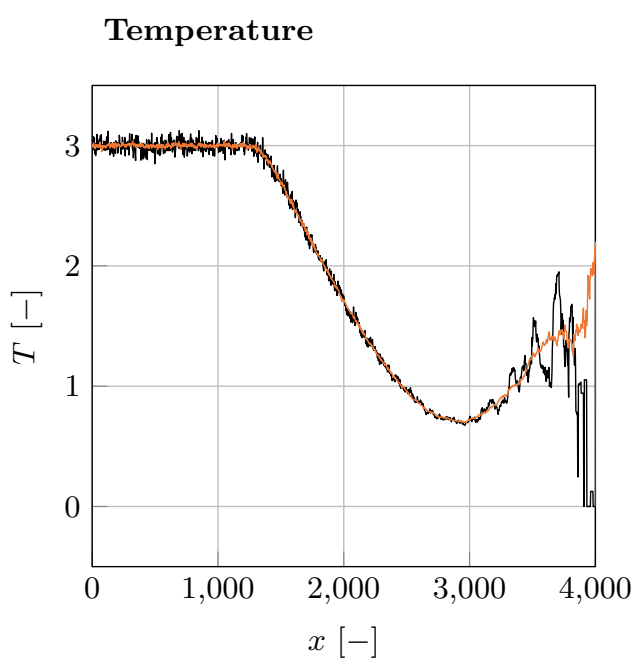

Figure 7: MD simulation of the vacuum Riemann problem VP1 at time $t=45.59$ for two different numbers of particles.

\subsubsection{Results for VP2 and VP3}

For the next two vacuum Riemann problems only results at time $t=$ 273.55 are shown. VP2 has the initial density $\rho_{L}=0.01$. The results are shown in Fig. 8, VP3 hast the initial density $\rho_{L}=0.1$. The results are shown in Fig. 9. The qualitative behavior is similar to the results for VP1. In the case of VP2 with the smaller density, the noise in the MD data increased, which is caused by the smaller number of particles per volume.

\subsubsection{Results for VP4}

The vacuum Riemann problem VP4 has a larger density of $\rho=0.2$. For this case, the expansion is much stronger than in the previous cases and leads to a sooner crossing of the isentropic expansion and the vapour binodal curve. Figure 10 shows the loci of constant entropy for all vacuum Riemann prob- 

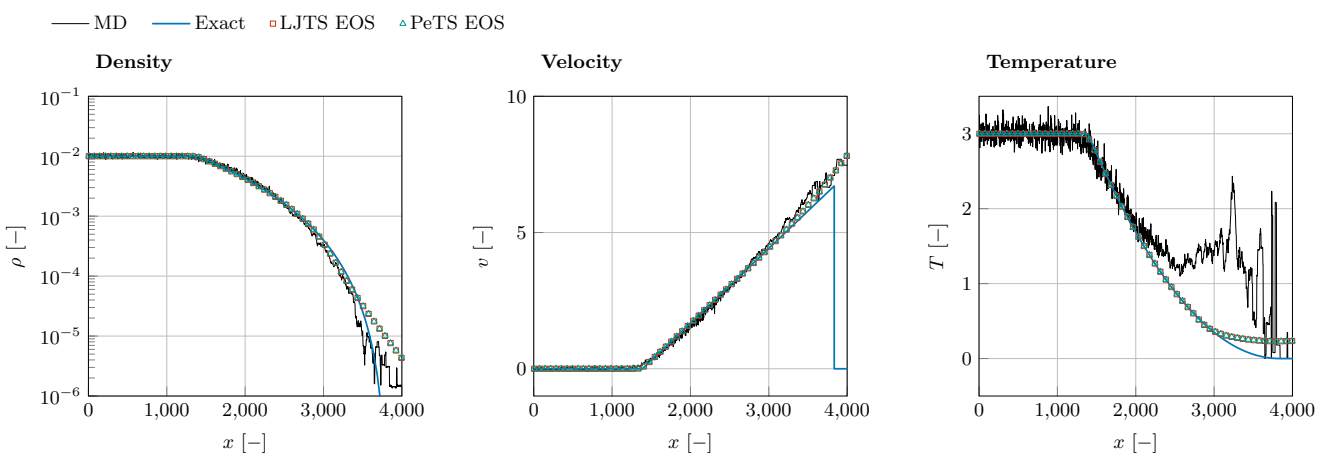

Figure 8: Results for the vacuum Riemann problem VP2 at time $t=273.55$. Shown is the CFD solution based on the LJTS EOS, the exact solution for the perfect gas EOS with $\gamma=1.667$ and the MD simulation results.
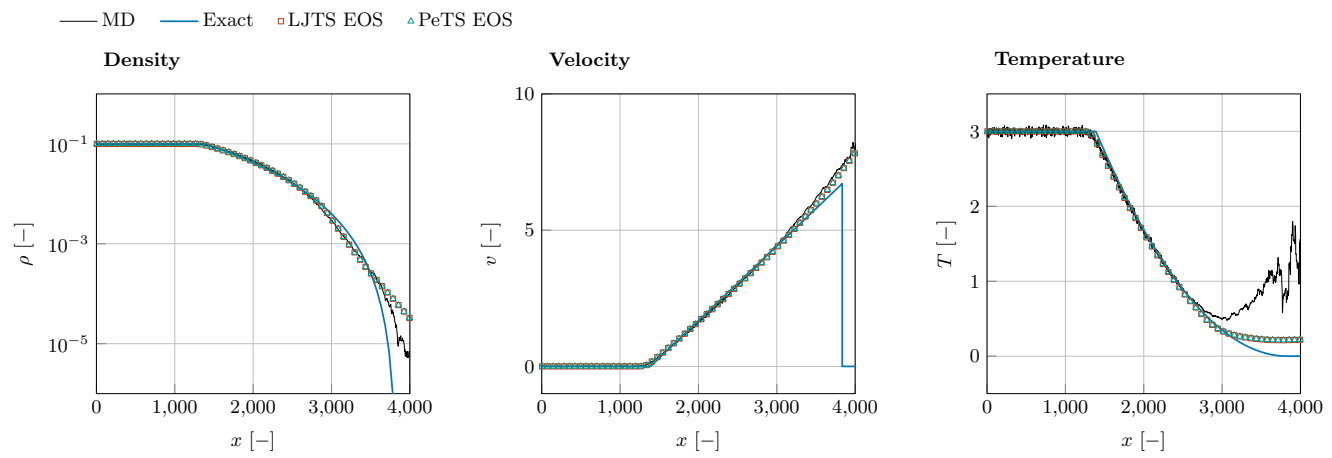

Figure 9: Results for the vacuum Riemann problem VP3 at time $t=273.55$. Shown is the CFD solution based on the LJTS EOS, the exact solution for the perfect gas EOS with $\gamma=1.667$ and the MD simulation results.

lems in state space. Eventually, most of the curves cross the vapour binodal, but only for the largest density droplets appeared. The results for density, velocity and temperature are presented in Fig. 11 at time $t=300.91$. Similar to the other scenarios, the results of the different methods are in very good agreement. The LJTS and PeTS EOS allow a robust simulation in this regime, in which small droplets in the expansion fan occur by accounting for 


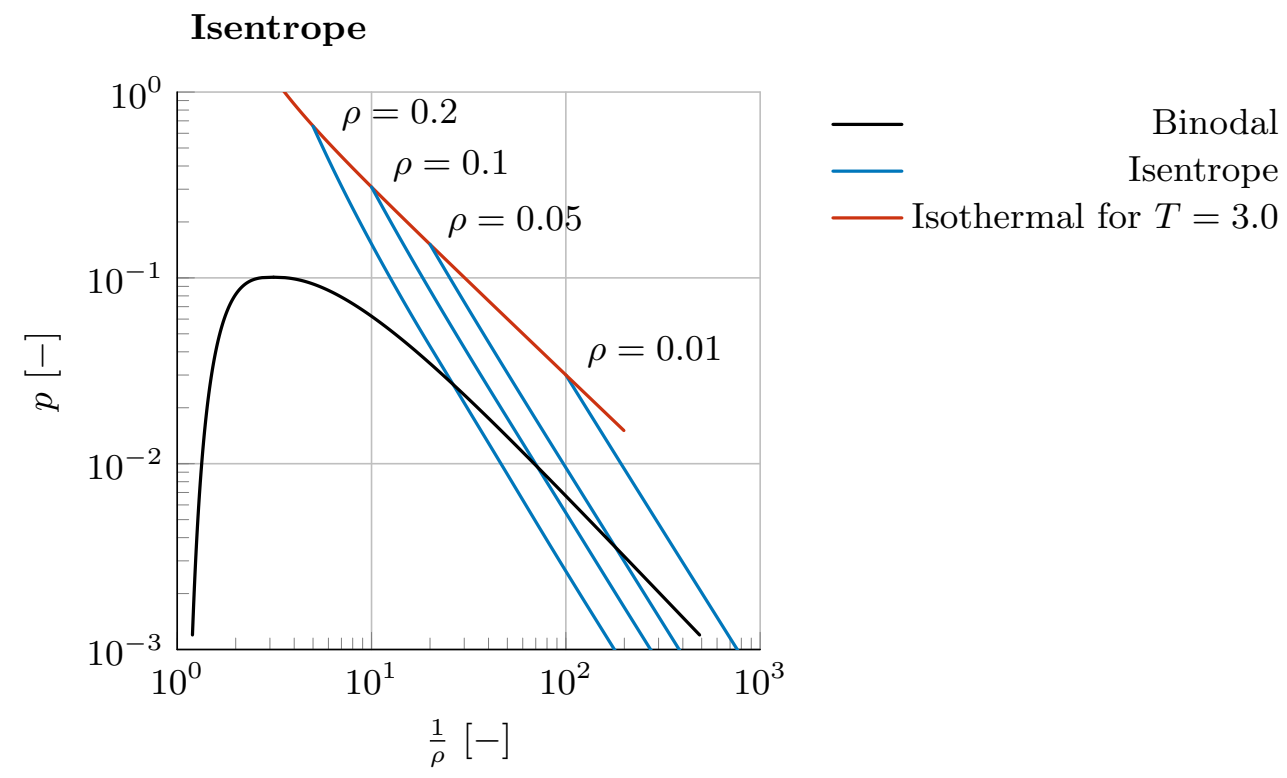

Figure 10: Isentropic expansion curves in state space for the vacuum Riemann problems VP1-VP4.

a undercooled vapour. Due to the fact that the phase change is not explicitly included into the simulation and the equation of state, the CFD simulations break down if the initial density is further increased. In the MD simulations, the droplets grew in size. In this case the strong isentropic expansion moves into the region of the LJTS fluid, where both fail to provide macroscopic physical states. This is the region, in which the underlying pressure function becomes non-convex and the speeds of sound become imaginary. In these cases, a phase transition model has to be introduced and the multiphase Riemann problem changes its structure taking into account the phase transition line. 

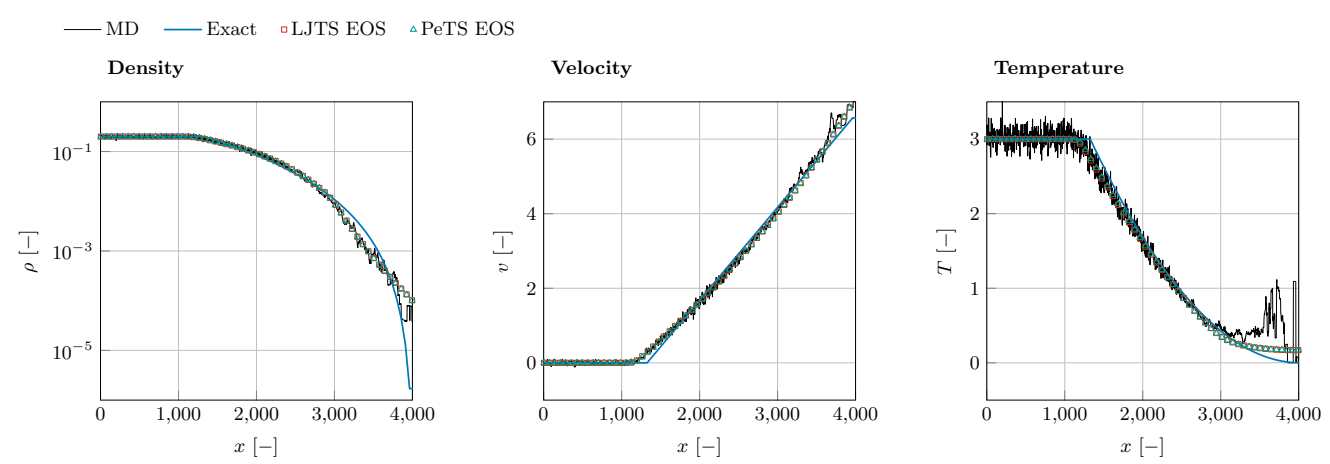

Figure 11: Results for the vacuum Riemann problem VP4 at time $t=300.91$. Shown is the CFD solution based on the LJTS EOS, the exact solution for the perfect gas EOS with $\gamma=1.667$ and the MD simulation results.

\section{Conclusion}

We compared the macroscopic Riemann solution and approximate solutions, obtained by a CFD solver, with microscopic solutions, obtained by molecular dynamic simulations. We picked up two Riemann problem scenarios. The numerical results can be directly compared because both methods are based on a Lennard-Jones model fluid with truncated and shifted potential. The MD simulations use this potential, for the CFD simulation the corresponding accurate equations of state were used, proposed in Thol et al. [5] and in Heier et al. [6]. The CFD simulations were based on a hybrid DG/FV method with the Suliciu relaxation solver as numerical flux function. The two scenarios under investigation were a super-critical shock tube problem and a strong expansion of a gas into vacuum. The agreement between the macroscopic and microscopic solutions is nearly perfect. In the super-critical regime, the underlying EOS were able to reproduce liquid-like and vapour-like states and their transition from one to the other regime. 
In the second problem, the vacuum Riemann problem, the EOS approaches the regime of a perfect gas. Hence, the exact solution for perfect gas law coincides very well with the results. Four test cases with varying initial densities have been considered. The agreement with the microscopic solutions was very good. Beyond the critical Knudsen number, the microscopic solution developed non-equilibrium states, where a continuum temperature is not well-defined any more. Hence, in the low density region, the macroscopic and microscopic solutions differed. By increasing the strength of the expansion in the vacuum Riemann problem, condensation starts and small liquid droplets appeared in the expansion fan simulated by molecular dynamics. The real gas EOS could reproduce the solution as long as this effect could be approximated as undercooled vapour phase. A further increase of the strength of the expansion wave lead to a failure and a break down of the continuum simulations. Hence, for stronger rarefaction waves, the phase transition has to be explicitly modelled in the numerical approach.

These results strongly motivate the use of MD simulations as comparison in the cases with phase transitions. The MD results may establish thermodynamic consistent solutions for benchmark problems that can be used as benchmarks for macroscopic multiphase flow approximations. As mentioned in the introduction, our research activities are embedded in the DFG collaborative research center SFB-TRR 75 "Droplets under extreme ambient conditions" and we are able to continue this research in the forthcoming years. MD results for these situations with phase transitions will be well documented. 


\section{Acknowledgements}

The work was supported by the German Research Foundation (DFG) through SFB-TRR 75 "Droplet Dynamics under Extreme Ambient Conditions". The MD simulations were performed on the national supercomputer Cray XC40 (Hazel Hen) at the High Performance Computing Center Stuttgart (HLRS).

\section{References}

[1] S. K. Godunov, A difference method for numerical calculation of discontinuous solutions of the equations of hydrodynamics, Mat. Sb. (N.S.) 47 (1959) 271-306.

[2] E. F. Toro, Riemann solvers and numerical methods for fluid dynamics: a practical introduction, 2 ed., Springer Science \& Business Media, Berlin, Heidelberg, 2009.

[3] R. Menikoff, B. J. Plohr, The Riemann problem for fluid flow of real materials, Reviews of Modern Physics 61 (1989) 75-130.

[4] P. G. LeFloch, Hyperbolic Systems of Conservation Laws, Lectures in Mathematics. ETH Zürich, 1 ed., Birkhäuser, Basel, 2002.

[5] M. Thol, G. Rutkai, R. Span, J. Vrabec, R. Lustig, Equation of State for the Lennard-Jones Truncated and Shifted Model Fluid, International Journal of Thermophysics 36 (2015) 25-43. doi:10.1007/s10765014-1764-4. 
[6] M. Heier, S. Stephan, J. Liu, W. G. Chapman, H. Hasse, K. Langenbach, Equation of state for the Lennard-Jones truncated and shifted fluid with a cut-off radius of 2.5 sigma based on perturbation theory and its applications to interfacial thermodynamics, Molecular Physics 116 (2018) 2083-2094.

[7] O. Kunz, W. Wagner, The GERG-2008 wide-range equation of state for natural gases and other mixtures: an expansion of GERG-2004, Journal of Chemical \& Engineering Data 57 (2012) 3032-3091.

[8] O. Kunz, R. Klimeck, W. Wagner, M. Jaeschke, The GERG-2004 widerange equation of state for natural gases and other mixtures, Ber. VDI, VDI-Verlag, Duesseldorf (2007).

[9] R. Span, Multiparameter Equations of State, Springer-Verlag, Berlin, Heidelberg, 2000.

[10] J. A. Barker, D. Henderson, Perturbation Theory and Equation of State for Fluids: The SquareWell Potential, The Journal of Chemical Physics 47 (1967) 2856-2861. doi:10.1063/1.1712308.

[11] P. Colella, H. M. Glaz, Efficient solution algorithms for the Riemann problem for real gases, Journal of Computational Physics 59 (1985) 264-289. doi:10.1016/0021-9991(85)90146-9.

[12] J. R. Kamm, An Exact, Compressible One-Dimensional Riemann Solver for General, Convex Equations of State, Technical Report LA-UR-1521616, Los Alamos National Laboratory, 2015. 
[13] E. Halter, E. Martensen, A fast solver for Riemann problems, Mathematical Methods in the Applied Sciences 7 (1985) 101-107. doi:10.1002/mma.1670070107.

[14] T.-P. Liu, J. Smoller, On the vacuum state for the isentropic gas dynamics equations, Advances in Applied Mathematics 1 (1980) 345-359. doi:10.1016/0196-8858(80)90016-0.

[15] C.-D. Munz, A tracking method for gas flow into vacuum based on the vacuum Riemann problem, Mathematical Methods in the Applied Sciences 17 (1994) 597-612. doi:10.1002/mma.1670170803.

[16] F. Hindenlang, G. J. Gassner, C. Altmann, A. Beck, M. Staudenmaier, C.-D. Munz, Explicit discontinuous Galerkin methods for unsteady problems, Computers \& Fluids 61 (2012) 86-93. doi:10.1016/j.compfluid.2012.03.006.

[17] M. Sonntag, C.-D. Munz, Shock Capturing for Discontinuous Galerkin Methods using Finite Volume Subcells, in: J. Fuhrmann, M. Ohlberger, C. Rohde (Eds.), Finite Volumes for Complex Applications VII-Elliptic, Parabolic and Hyperbolic Problems, Springer International Publishing, 2014, pp. 945-953.

[18] M. Sonntag, C.-D. Munz, Efficient Parallelization of a Shock Capturing for Discontinuous Galerkin Methods using Finite Volume Sub-cells, Journal of Scientific Computing 70 (2017) 1262-1289. doi:10.1007/s10915-016-0287-5. 
[19] P.-O. Persson, J. Peraire, Sub-Cell Shock Capturing for Discontinuous Galerkin Methods, in: Proceedings of the 44th AIAA Aerospace Sciences Meeting and Exhibit, American Institute of Aeronautics and Astronautics, Reno, 2006.

[20] C. A. Kennedy, M. H. Carpenter, R. Lewis, Low-storage, explicit Runge-Kutta schemes for the compressible Navier-Stokes equations, Applied Numerical Mathematics 35 (2000) 177-219. doi:10.1016/S01689274(99)00141-5.

[21] I. Suliciu, On modelling phase transitions by means of rate-type constitutive equations. Shock wave structure, International Journal of Engineering Science 28 (1990) 829-841. doi:10.1016/0020-7225(90)90028-H.

[22] I. Suliciu, Some stability-instability problems in phase transitions modelled by piecewise linear elastic or viscoelastic constitutive equations, International Journal of Engineering Science 30 (1992) 483-494. doi:10.1016/0020-7225(92)90039-J.

[23] F. Bouchut, Nonlinear Stability of Finite Volume Methods for Hyperbolic Conservation Laws, Frontiers in Mathematics, 1 ed., Birkhäuser, Basel, 2004.

[24] Z. Shen, W. Yan, G. Yuan, A robust and contact resolving Riemann solver on unstructured mesh, Part II, ALE method, Journal of Computational Physics 268 (2014) 456-484. doi:10.1016/j.jcp.2014.03.003.

[25] C. Niethammer, S. Becker, M. Bernreuther, M. Buchholz, W. Eckhardt, A. Heinecke, S. Werth, H.-J. Bungartz, C. W. Glass, H. Hasse, J. Vrabec, 
M. Horsch, ls1 mardyn: The Massively Parallel Molecular Dynamics Code for Large Systems, Journal of Chemical Theory and Computation 10 (2014) 4455-4464. doi:10.1021/ct500169q.

[26] J. Vrabec, G. K. Kedia, G. Fuchs, H. Hasse, Comprehensive study of the vapour-liquid coexistence of the truncated and shifted LennardJones fluid including planar and spherical interface properties, Molecular Physics 104 (2006) 1509-1527. doi:10.1080/00268970600556774.

[27] G. Rutkai, M. Thol, R. Span, J. Vrabec, How well does the Lennard-Jones potential represent the thermodynamic properties of noble gases?, Molecular Physics 115 (2017) 1104-1121. doi:10.1080/00268976.2016.1246760.

[28] I. D. Boyd, G. Chen, G. V. Candler, Predicting failure of the continuum fluid equations in transitional hypersonic flows, Physics of Fluids 7 (1995) 210-219. doi:10.1063/1.868720.

[29] G. Bird, Molecular Gas Dynamics and The Direct Simulation of Gas Flow, Oxford University Press, Oxford, 1994.

[30] J. M. Burt, I. D. Boyd, A hybrid particle approach for continuum and rarefied flow simulation, Journal of Computational Physics 228 (2009) 460-475. doi:10.1016/j.jcp.2008.09.022. 